\section{Host-Microbe Interactions}

\section{6-20 November 2013, Marrakech, Morocco}

The 14th IUBMB Conference, on the theme of "HostMicrobe Interactions" is to be held at the Palmeraie Conference Center in Marrakech, Morocco, from 16-20 November 2013. The conference will provide a forum for intensive scientific exchanges on the latest research and best practices around molecular life sciences.

IUBMB is also pleased to encourage you and your colleagues to take advantage of this unique opportu- nity to share and discuss your expertise and research in Host-Microbe Interactions with your peers. Here are the categories your abstract should fall under:

- host-pathogen interactions

- host defense mechanisms

- toxins

- bacterial resistance

- host-microbe coexistence-the microbiome

www.iubmb-2013.org

\section{Molecular Order and Mobility in Polymer Systems}

\section{2-6 June 2014, St. Petersburg, Russia}

The 8th International Symposium on Molecular Order and Mobility in Polymer Systems will be held in St. Petersburg, Russia, 2-6 June 2014. The main aim of the symposium is to discuss the modern problems of physics and chemistry of complex stimuli-responsive polymer systems with nano-structure organization and "soft" order preserving a pronounced molecular mobility. Both the experimental data and the results of theory and simulation will be presented. The main focus of the symposium will be the equilibrium properties of polymer systems.
Symposium topics include the following:

1. conformations and properties of macromolecules of complex architecture: star-like molecules, molecular brushes, dendrimers

2. structure and properties of polymer brushes and haired nano-objects

3. macromolecular self-assembly: micelles, multicompartment structures, inter-polymer/polyelectrolyte complexes

4. structure and properties of complexes of polymers with nano-particle or biological object

5. polymer networks and composites

www.macro.ru

\section{Polymer-Solvent Complexes and Intercalates}

22-25 September 2014, Salerno, Italy

The 10th International IUPAC Conference on Polymer-Solvent Complexes and Intercalates, will be held in Salerno, Italy, from 22-25 September 2014. POLYSOLVAT-10 will be the latest in a series of successful meetings organized every two years, the most recent of which were held in Kiev (2012), Strasbourg (2010), and Marrakech (2008).

The conference will focus on the formation mechanisms, morphology, molecular structure, and properties of compounds from synthetic polymers, biopolymers, proteins, and supramolecular polymers. The bulk state, the solutions and the systems formed at surfaces/ interfaces will be considered.

The program for the conference will consist of 10 invited lectures, about 20 oral communications, and 2 poster sessions, which will provide participants the opportunity to highlight their recent work. The conference proceedings will be published in a special issue of Macromolecular Symposia.

The conference will be organized and hosted by Christophe Daniel and Gaetano Guerra of the Dipartimento di Chimica e Biologia, Università degli Studi di Salerno.

wiil ww.polysolvat10.unisa.it 